\title{
Croissance de l'épicéa, du mélèze, du pin cembro et du pin à crochets en limite supérieure de la forêt dans quatre régions des Alpes françaises
}

\author{
V Petitcolas*, C Rolland, R Michalet \\ Laboratoire des écosystèmes alpins, Centre de biologie alpine, université Joseph-Fourier, \\ BP 53 X, 3804I Grenoble cedex 9, France
}

(Reçu le 10 février 1997 ; accepté le 3 juin 1997)

\begin{abstract}
Summary - Tree-growth of spruce, larch, arolla pine and mountain pine near the timberline in four french alpine areas. The radial growth and the dominant height of the Norway spruce, the European larch, the arolla pine and the mountain pine growing near the timberline are analysed in four areas located in the French Alps (Briançonnais, Haute-Maurienne, Moyenne-Tarentaise and Belledonne), on two exposures (northern and southern slopes) and two kinds of bedrock (calcareaous and non-calcareaous). The radial growth is globally higher in the Moyenne-Tarentaise, and lower in the Briançonnais. The radial growth of the spruce and the arolla pine is relatively high, particularly on noncalcareous bedrocks and southern slopes, whereas that of the mountain pine and the larch is lower and less dependant on stational conditions. The rhythms of growth expressed in basal area increment also differ, with a more extended period of high growth rate for the arolla pine compared to the spruce, a very slow decline of the ring areas of the mountain pine after 75 years and the stabilisation of those of the larch between at least 75 and 350 years. Finally, the spruce and the larch show the best dominant heights.
\end{abstract}

Norway spruce / European larch / arolla pine / mountain pine / growth / subalpine

Résumé - La croissance radiale et la hauteur dominante de l'épicéa, du pin cembro, du mélèze et du pin à crochets poussant en limite supérieure de la forêt ont été étudiées dans quatre régions bioclimatiques des Alpes françaises (Briançonnais, Haute-Maurienne, Moyenne-Tarentaise et Belledonne), dans deux expositions (adret et ubac) et sur deux types de roche-mère (calcaires et non carbonatées). La croissance radiale en fonction de l'âge est la plus faible dans le Briançonnais et la plus forte en Moyenne-Tarentaise. Les comportements de l'épicéa et du pin cembro sont assez proches, avec une croissance radiale relativement forte (rayon moyen de $20 \mathrm{~cm}$ à 150 ans), particulièrement sur substrats silicatés et en adret, tandis que celle du mélèze et du pin à crochets est plus faible (rayon

\footnotetext{
* Correspondance et tirés à part

Tél : (33) 0476514680 ; fax : (33) 0476514463 ; courriel : remi.foussadier@ujf-grenoble.fr
} 
moyen de $20 \mathrm{~cm}$ atteint 100 ans plus tard) et moins dépendante des conditions stationnelles. Les rythmes de croissance exprimés en surface terrière diffèrent également : phase d'accroissement soutenu plus longue pour l'épicéa que pour le pin cembro, diminution très progressive de la croissance du pin à crochets après 75 ans, âge à partir duquel la croissance du mélèze présente un pallier très stable se poursuivant jusqu'à au moins 350 ans. Enfin, le mélèze et l'épicéa présentent les plus fortes hauteurs dominantes.

épicéa / mélèze / pin cembro / pin à crochets / croissance / étage subalpin

\section{INTRODUCTION}

La croissance des arbres poussant en limite supérieure de la forêt ainsi que dans la zone de combat subalpine a été encore peu étudiée en France d'un point de vue dendrométrique, ces forêts étant généralement considérées plus comme des forêts de protection que de production à cause de leurs productivités médiocres (Trosset, 1984).

Il est pourtant intéressant d'analyser la croissance de ces arbres (Gil-Pelegrin et Villar-Perez, 1988), car ils poussent dans des conditions extrêmes qui caractérisent leurs limites écologiques (Arquillière, 1986). Les conifères présents à haute altitude ont d'ailleurs fait l'objet de plusieurs études dendroécologiques (Matthewes, 1976), parfois de façon comparative en particulier dans les Alpes (Tessier, 1981 ; Siebenlist-Kerner, 1984 ; Petitcolas, 1993) mais le plus souvent séparément. Ainsi, dans l'étage subalpin des Alpes françaises, Contini et Lavarello (1982) se sont intéressés au pin cembro, Serre (1978), au mélèze, et Rolland et Schueller (1995, 1996), au pin à crochets et à l'épicéa. De tels sites ont également donné lieu à des études concernant l'écologie (Fourchy, 1952) et l'écophysiologie des espèces (Tranquillini, 1979). Ces recherches permettent une meilleure connaissance des limites des potentialités des essences forestières et peuvent s'avérer utiles pour le choix d'essences lors de reboisements (Mullenbach, 1982), par exemple pour la lutte contre les avalanches.

Notre objectif est ici d'étudier, à partir de mesures de largeurs de cernes et de hau- teurs dominantes, les quatre principaux conifères capables de pousser à haute altitude dans les Alpes françaises : l'épicéa (Picea abies (L) Karst), le mélèze (Larix decidua Mill), le pin cembro (Pinus cembra L) et le pin à crochets (Pinus uncinata Mill ex Mirb). Dans un premier temps, la croissance radiale des arbres en fonction de l'âge cambial est analysée dans quatre régions différentes en recherchant, pour chaque essence, une éventuelle influence de la roche-mère et de l'exposition. Dans un deuxième temps une analyse par essence est réalisée en regroupant, pour chacune d'elles, toutes les courbes disponibles, afin de comparer leurs dynamiques spécifiques. La prise en compte de la hauteur moyenne des arbres permet enfin de compléter les résultats relatifs à la croissance radiale.

\section{MATÉRIEL ET MÉTHODES}

\section{Les sites d'étude}

Les stations étudiées se répartissent dans quatre régions bioclimatiques des Alpes françaises (figure 1) définies par trois facteurs principaux. Le premier facteur concerne l'aridité estivale, exprimée par l'angle de Gams estival (Michalet, 1991 ; Pache et al, 1996), d'autant plus fort que les étés sont secs. Le second facteur concerne les précipitations hivernales : il représente la continentalité hydrique, exprimée par l'angle de Gams hivernal, d'autant plus faible que le climat est océanique. Dans la zone étudiée, les angles de Gams estivaux et hivernaux varient dans le même sens, traduisant l'augmentation concomitante de l'aridité estivale et de la continentalité hydrique en allant des Alpes externes aux Alpes internes. Le troisième facteur est un 


\section{Chantelouve}
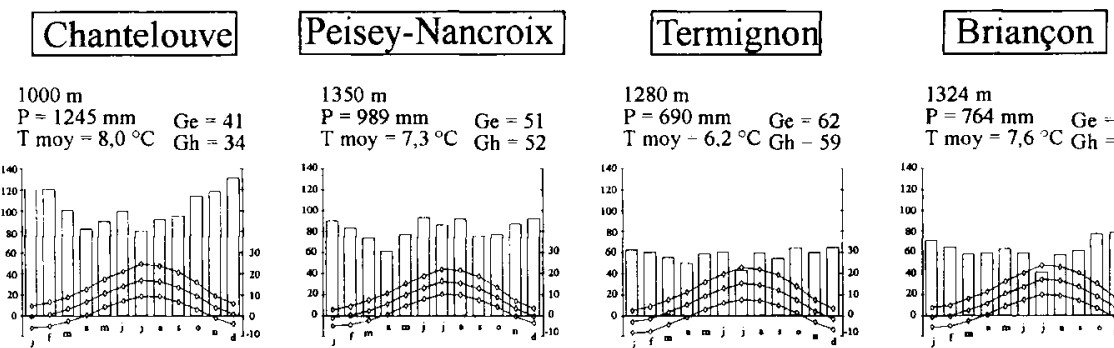

période

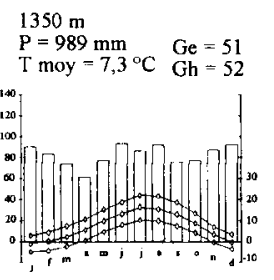

période 1951-90

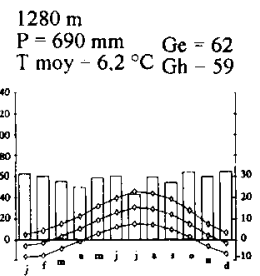

période 1951-90
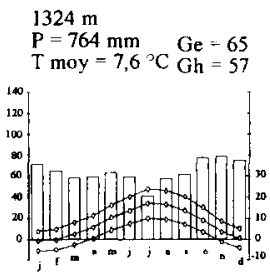

période 1951-90

$1951-90(\mathrm{P})$ et 1967-90 (T)

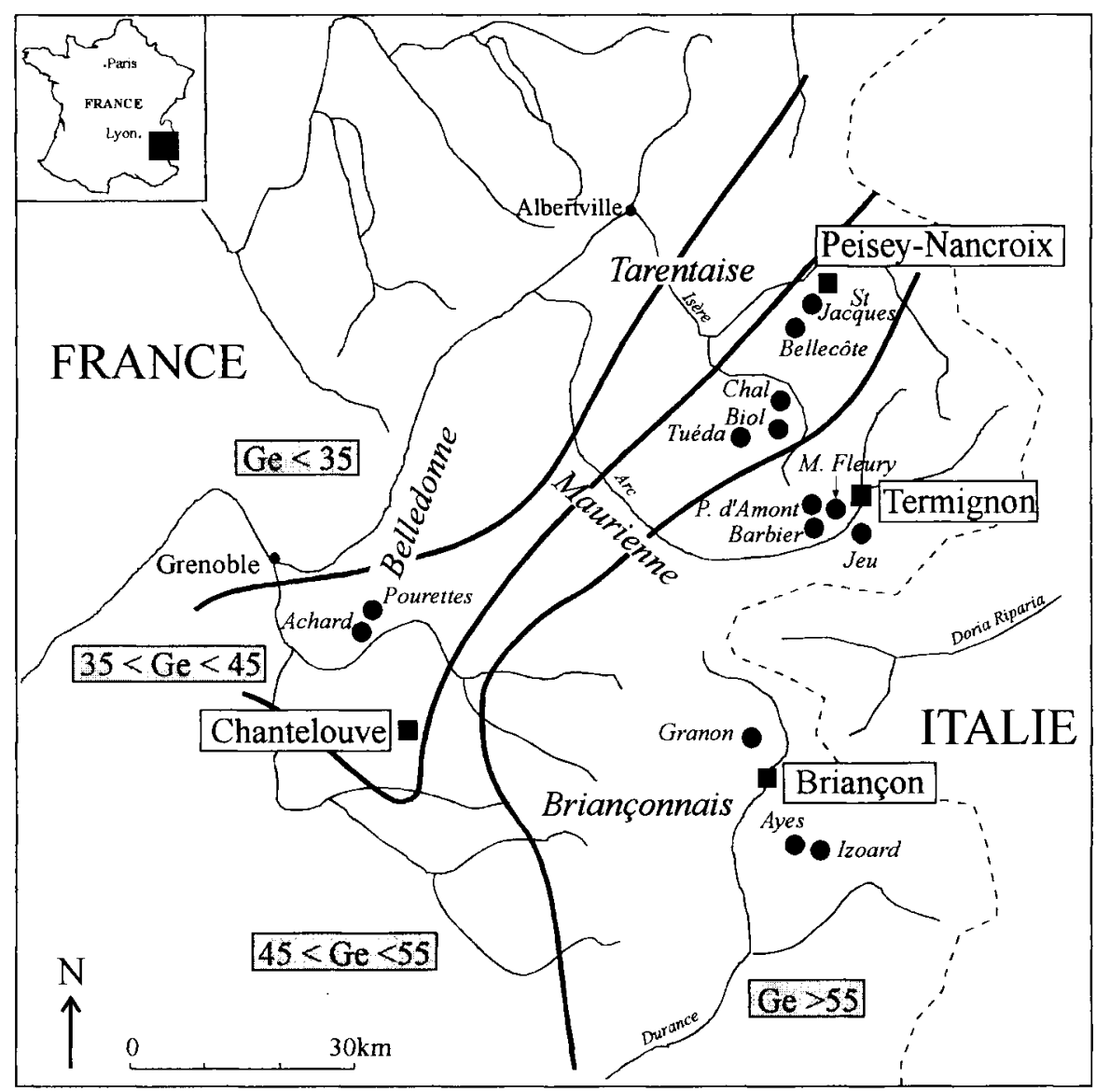

Site d'étude

Station météorologique

Fig 1. Position géographique des sites étudiés, diagrammes ombrothermiques des stations météorologiques proches et zonation bioclimatique en fonction des angles de Gams estivaux. 
facteur thermique : il distingue, parmi les stations de la zone interne (angles de Gams élevés), celles du nord, plus fraîches, de celles du sud, plus chaudes. Les sites de prélèvements se trouvent dans les régions suivantes, situées entre $44^{\circ} 48^{\prime}$ et $45^{\circ} 30^{\prime}$ 'de latitude Nord et de $5^{\circ} 54^{\prime}$ à $6^{\circ} 48^{\prime}$ de longitude Est.

- Le Briançonnais, dans les Alpes internes sudoccidentales à été sec et chaud, où l'épicéa est exclu par la sécheresse estivale.

- La Haute-Maurienne, dans les Alpes internes nord-occidentales, à été aussi sec mais aux températures plus fraîches que celles du Briançonnais.

- La Moyenne-Tarentaise, dans les Alpes intermédiaires nord-occidentales, à été assez bien arrosé.

- Le massif de Belledonne, dans les Alpes externes nord-occidentales mieux arrosées, où le mélèze est absent à cause du caractère trop océanique du climat.

Dans chacune de ces régions, les stations sont localisées sur deux types de roches mères (calcaires et non carbonatées) et réparties selon deux expositions (adret et ubac), soit quatre modalités écologiques par région climatique (deux seulement pour le massif cristallin de Belledonne où toutes les stations se trouvent sur roches mères non carbonatées) et 14 au total. Un seul niveau moyen d'altitude est analysé, celui de la limite forestière, variable ici de 1750 à $2300 \mathrm{~m}$ mais en général situé vers 2000 à $2100 \mathrm{~m}$. Il s'agit plus de la limite supérieure de la forêt que de celle des arbres isolés. La localisation ainsi que le descriptif détaillé des 37 populations étudiées sont résumés dans le tableau I.

L'échantillonnage a été réalisé dans un but avant tout dendroécologique, donc en favorisant la diversité écologique des lieux de prélèvement plus que le nombre d'échantillons par population. Il en découle l'impossibilité de calculer certains paramètres dendrométriques (par exemple la hauteur des arbres à 100 ans), et implique de rester prudent quant à l'interprétation et la généralisation des résultats.

Seules les situations les plus courantes au regard des quatre modalités écologiques envisagées ont été étudiées pour chaque essence, c'est pourquoi il manque parfois certaines combinaisons de conditions écologiques.

\section{Échantillonnage et données numériques}

Pour chacune des 37 populations, 12 arbres dominants ou codominants relativement à chaque essence ont été carottés à couur, à 1,3 m du sol et selon trois directions (une carotte en amont et deux de part et d'autre du tronc perpendiculairement à la pente). Les arbres dont le cour s'est révélé pourri au sondage ont été écartés. Chaque station de prélèvement a été décrite selon des critères floristiques et géologiques (tableau I) détaillés ici avant tout pour permettre de contrôler leur représentativité. L'ouverture du peuplement a été estimée (pourcentage de recouvrement de la strate arborescente) afin de rendre compte des phénomènes de compétition.

Les largeurs de cernes des 1320 carottes ont ensuite été mesurées au laboratoire avec une précision théorique de $0,01 \mathrm{~mm}$ à l'aide d'une chaîne de lecture informatisée.

À partir des mesures brutes des largeurs de cernes (185 667 au total), deux paramètres représentatifs de la croissance radiale ont été calculés successivement pour chaque population (Rolland et Schueller, 1995) :

- le rayon moyen en fonction de l'âge courant (âge cambial) obtenu par le cumul des largeurs moyennes des cernes successifs,

- l'accroissement moyen de la surface terrière par l'âge courant, obtenu à partir des rayons moyens.

\section{RÉSULTATS ET DISCUSSION}

\section{Croissance radiale dans différentes conditions stationnelles}

Les courbes des rayons moyens par âge courant des 37 populations sont analysées dans chacune des quatre régions en recherchant, pour chaque essence, l'influence de la roche mère et de l'exposition (fig 2). Dans l'ensemble, le rayon à 200 ans est souvent compris entre 10 et $20 \mathrm{~cm}$ et n'atteint que très rarement $30 \mathrm{~cm}$.

\section{Briançonnais}

L'épicéa étant très rare dans le Briançonnais, seules les trois autres essences y ont 


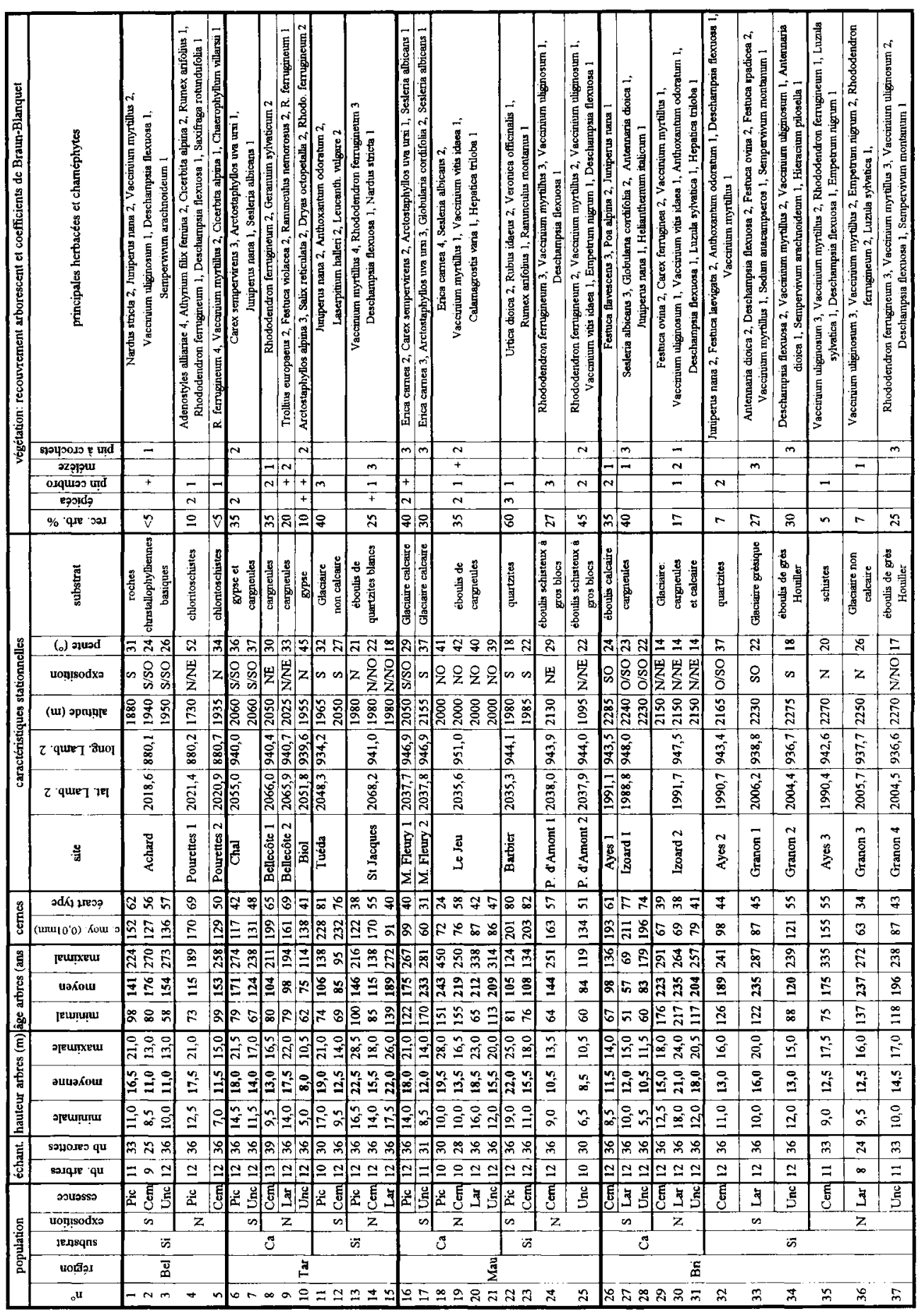

ฮี
0
0
0
0
0
0

ชิ

를

응

要的

ప

क्ष

记

일

플

药

बิ

造要

․․ $>$

氖

舫

要

正

守

를

过:

它敦

它它

䒠言

$\sum \hat{z}$

\%

跣

艺官

岂导

도용

क्जे

要

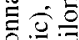

这

两

谷:

$\ddot{0}$

$. \bar{z}=$

흥 홍

․

$8 \frac{0}{2}$

8 


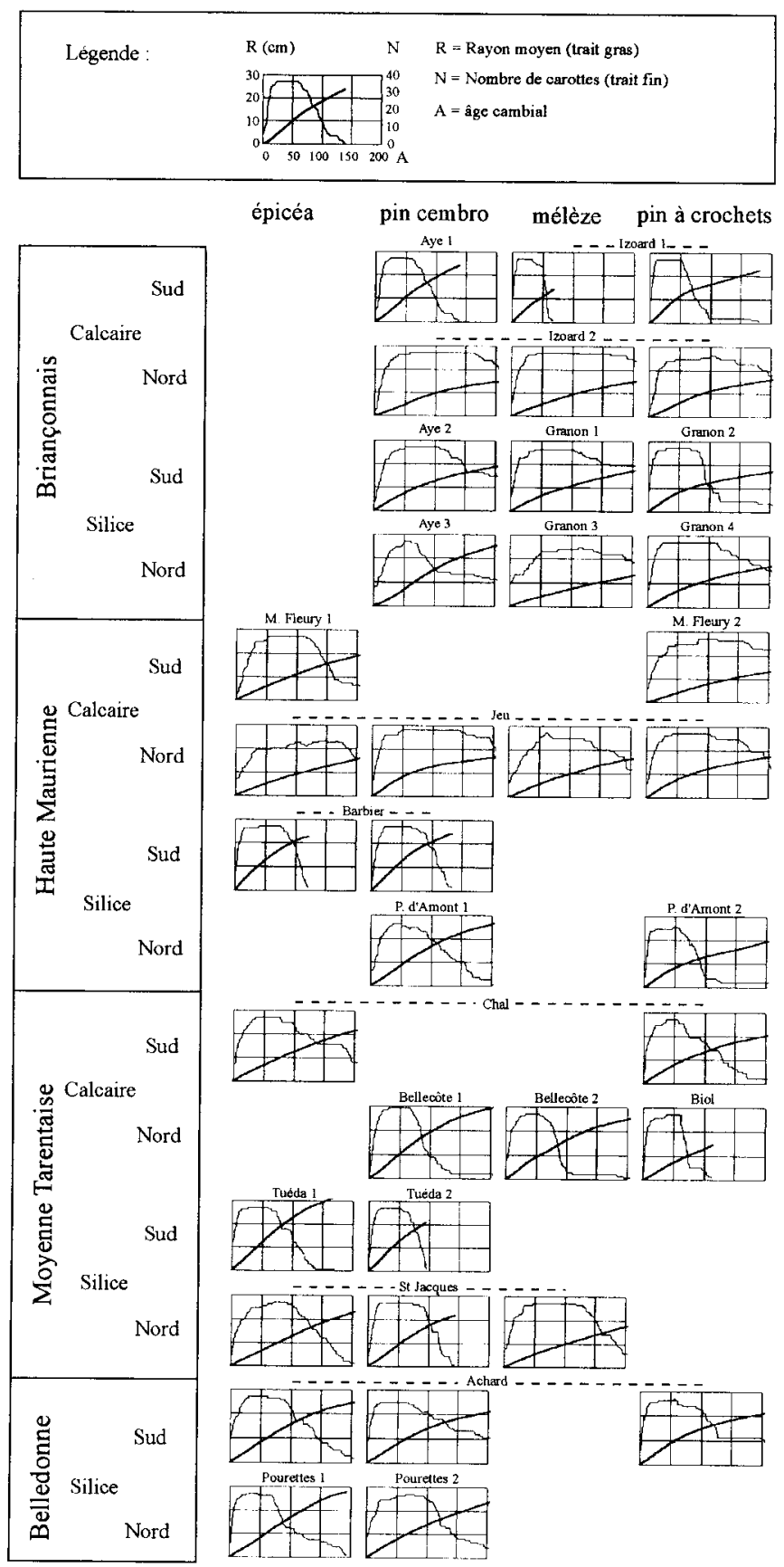

Fig 2. Rayons moyens des troncs ( $\mathrm{en} \mathrm{cm}$ ) en fonction de l'âge courant pour les 37 populations réparties par région, par type de roche mère et par exposition. 
été échantillonnées et les quatre types de biotope sont représentées pour chacune d'elles.

- Le pin cembro pousse le mieux en adret sur roche calcaire, avec un rayon à 100 ans $\left(\mathrm{R}_{100}\right)$ proche de $20 \mathrm{~cm}$, et le moins bien en ubac sur roche calcaire avec un $R_{100}$ de 10 $\mathrm{cm}$. La présence de nombreuses souches dans la station d'ubac témoigne d'une compétition importante et explique probablement en partie cette croissance plus faible.

- Le mélèze présente, lui aussi, une croissance nettement plus forte en adret sur roche calcaire, sa plus faible croissance étant observée en ubac sur roche décarbonatée. Cette différence de croissance selon l'exposition avait déjà été observée pour cette essence dans l'étage subalpin de cette région (Florence-Schueller, 1986).

- Le comportement du pin à crochets est très proche de celui du pin cembro et du mélèze, avec toujours une croissance plus forte en adret sur roche calcaire. On ne retrouve pas une meilleure croissance en adret sur roche silicatée, alors que FlorenceSchueller et Rolland (1995) l'avaient observée dans l'étage subalpin des Pyrénées orientales françaises. Nos résultats ne montrent pas non plus de différence nette de croissance selon la roche mère, alors qu'une étude réalisée dans l'étage subalpin du Briançonnais et du Queyras a montré une croissance radiale supérieure de 18,6\% sur substrat calcaire par rapport aux substrats silicatés (Schueller et Rolland, 1994).

\section{Haute-Maurienne}

Les quatre essences étudiées sont présentes dans cette région, mais n'y ont été étudiées que dans les types de biotopes où elles sont suffisamment représentées.

La croissance de l'épicéa (trois populations) est plus faible en ubac sur roche calcaire $\left(\mathrm{R}_{100}<10 \mathrm{~cm}\right)$ et plus forte en adret sur roche silicatée $\left(\mathrm{R}_{100}>20 \mathrm{~cm}\right)$ dans le site du Barbier. Notons toutefois que la combinai- son ubac-roche silicatée n'est pas représentée et que le site du Barbier présente un sol profond, actif et riche en azote (végétation nitrophile), qui contribue probablement à favoriser la croissance, renforçant ainsi l'influence de l'exposition et de la roche mère.

Sur trois populations, le pin cembro se montre, comme l'épicéa et avec une croissance similaire, plus productif en adret sur roche silicatée (même site du Barbier). Pour un même type de station, il pousse mieux que dans le Briançonnais bien que les peuplements soient à la fois plus fermés et plus proches de la limite forestière.

Une seule population de mélèze a été échantillonnée en Haute-Maurienne, en ubac sur roche calcaire (station du Jeu). Il s'agit de la seule station de l'échantillonnage dans laquelle les quatre essences sont présentes en mélange. La croissance du mélèze y est assez faible $\left(R_{100}=10 \mathrm{~cm}\right)$, proche de celle de l'épicéa et légèrement inférieure à celle des pins surtout durant les 100 premières années de vie des arbres.

Le pin à crochets (trois populations) montre une croissance plus faible en adret sur roche calcaire $\left(\mathrm{R}_{100}=8 \mathrm{~cm}\right)$ alors que ce type de station était le plus productif dans le Briançonnais.

\section{Moyenne-Tarentaise}

Ici encore, on trouve les quatre essences mais avec, selon les espèces, une représentation plus ou moins incomplète des types de biotope.

Trois populations d'épicéa ont été échantillonnées. Celle située en adret sur roche silicatée est la plus productive de tout l'échantillonnage $\left(R_{100}=22,5 \mathrm{~cm} ; R_{150}=\right.$ $30 \mathrm{~cm}$ ), ce qui correspond au même type de station favorable qu'en Haute-Maurienne.

Pour le pin cembro, trois populations ont été échantillonnées également. Là encore, c'est celle située en adret sur roche silicatée qui présente la plus forte croissance. Pour 
un même type de station, le pin cembro semble pousser encore mieux ici qu'en Haute-Maurienne.

Nous ne disposons que de deux populations de mélèze, en ubac, la meilleure croissance étant observée sur roche calcaire.

Enfin, deux populations de pin à crochets ont été échantillonnées sur roches calcaires, présentant une croissance moyenne semblable quelle que soit l'exposition.

\section{Massif de Belledonne}

Dans ce massif cristallin externe, toutes les stations sont situées sur roches mères non carbonatées. Le mélèze, qui requiert un climat sec et lumineux (Fourchy, 1952), en est absent en raison des influences trop océaniques du climat. Le pin cembro arrive juste en limite occidentale de son aire à Chamrousse et l'épicéa trouve ici son extension maximale. La croissance de l'épicéa, du pin cembro et du pin à crochets (une seule population en adret pour ce dernier) est, bien que légèrement supérieure pour l'épicéa, assez semblable quelle que soit l'exposition et plutôt moyenne $\left(\mathrm{R}_{100}=15 \mathrm{~cm}\right)$. Ce n'est d'ailleurs que dans les conditions édaphiques les plus extrêmes que le pin à crochets pousse mieux que l'épicéa dans les Alpes externes, par exemple sur lapiaz à lithosol dans le Vercors (Rolland, Schueller, 1995).

\section{Croissance comparée des quatre essences}

\section{Croissance radiale}

Il est intéressant de réaliser un bilan général de la croissance radiale de chaque essence tous sites confondus, comme l'ont fait Becker (1987) et Bert (1992) pour le sapin des Vosges et du Jura ou Badeau (1995) pour le hêtre des plateaux calcaires de Lorraine, afin de comparer globalement la croissance de ces quatre conifères subalpins. De tels résultats ont déjà été obtenus pour l'épicéa
(Perrier in Bert, 1992 ; Desplanque, 1997) et pour le pin à crochets (Rolland, 1996). En revanche, le mélèze et le pin cembro n'ont pas encore, à notre connaissance, fait l'objet d'études au moyen de cette méthode.

L'homogénéité des courbes de croissance du pin à crochets d'une part et du mélèze d'autre part permet, pour chacune de ces deux essences, de regrouper les courbes de toutes les populations, quelles que soient la région considérée, la roche mère et l'exposition. Pour le pin cembro et l'épicéa, la tendance générale à une croissance plus forte sur roches silicatées nous a conduit à séparer les populations en deux sous groupes selon le type de roche-mère. Cette différence d'intensité de croissance selon la roche mère ressort de manière assez nette sur les courbes moyennes mais la forme des courbes, visiblement propre à l'espèce, reste la même pour une espèce donnée.

Les courbes des rayons moyens en fonction de l'âge courant (fig 3 ) font nettement ressortir la croissance radiale plus rapide de l'épicéa et du pin cembro qui atteignent dès 150 ans un rayon moyen de $20 \mathrm{~cm}$, alors qu'il faut 100 ans de plus au mélèze et au pin à crochets pour atteindre un même rayon.

Ces similitudes de croissance peuvent cependant être nuancées par l'examen des accroissements annuels de la surface terrière (fig 4). Seuls le pin cembro et l'épicéa présentent des accroissements moyens de la surface supérieurs à $10 \mathrm{~cm}^{2} / \mathrm{an}\left(7,5 \mathrm{~cm}^{2}\right.$ /an sur roches carbonatées pour l'épicéa). L'épicéa présente la période de croissance soutenue la plus longue, allant de 80 à 150 ans environ (200 ans sur roches carbonatées), avant de décliner de manière très progressive. En revanche le pin cembro montre un pallier de maturité plus court et un peu plus précoce, de 75 à 125 ans. Les similitudes apparentes de la croissance radiale de ces deux essences masquent donc des différences assez prononcées de dynamique de croissance. 
Epicéa

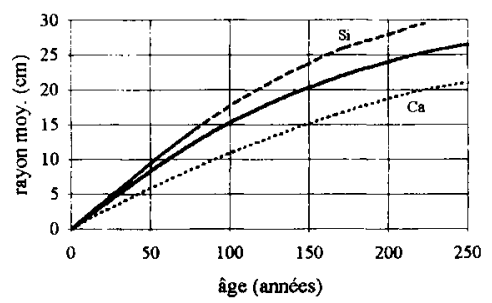

Pin cembro

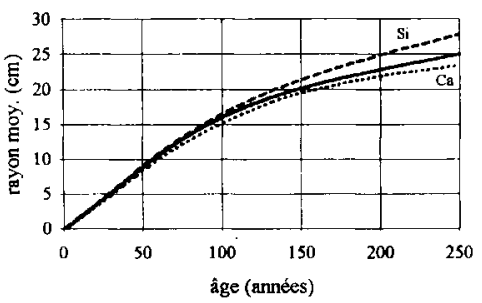

Mélèze

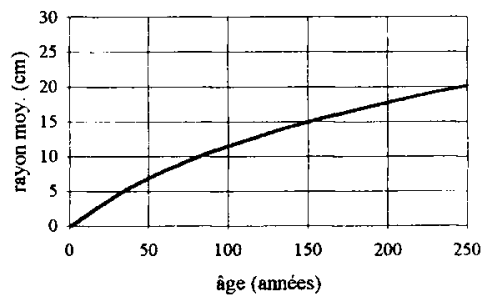

Pin à crochets

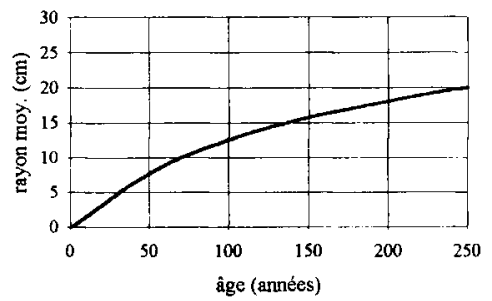

Fig 3. Rayons moyens des troncs (en $\mathrm{cm}$ ) en fonction de l'âge courant pour les quatre espèces, tous sites confondus $(-)$, sur roche-mères silicatées $(\mathrm{Si})$ et sur roches mères carbonatées $(\mathrm{Ca})$.

Epicéa

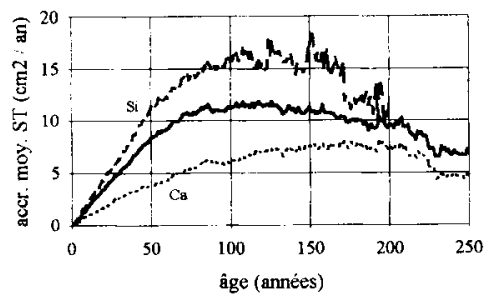

Pin cembro

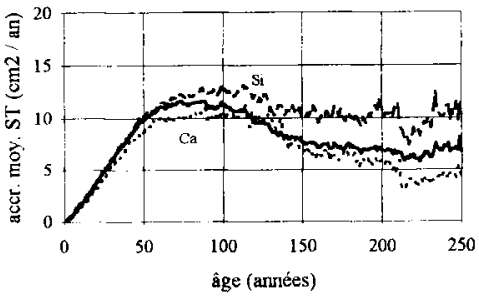

Mélèze

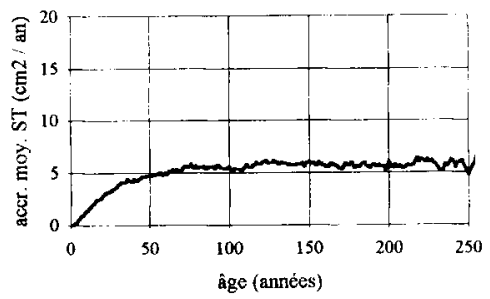

Pin a crochets

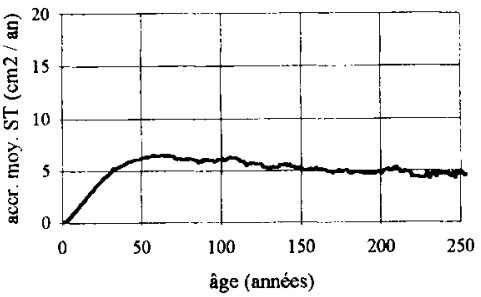

Fig 4. Accroissements moyens en surface terrière ( $\left(\mathrm{en}^{2} / \mathrm{an}\right)$ en fonction de l'âge courant pour les quatre espèces, tous sites confondus (-), sur roches mères silicatées ( $\mathrm{Si}$ ) et sur roches mères carbonatées $(\mathrm{Ca})$. 
Il en va de même pour le pin à crochets et le mélèze, à croissances radiales très comparables, mais dont les rythmes d'accroissement en surface terrière diffèrent assez nettement. Ainsi, le pin à crochets atteint son maximum d'accroissement vers 50 ans et voit sa croissance en surface réduire très graduellement dès 70 ans, alors qu'à partir de cet âge le mélèze présente un pallier continu très stable qui semble se prolonger extrêmement longtemps. Ce pallier a d'ailleurs été mis en évidence sur de longues chronologies d'arbres vivants (Tessier, 1986).

\section{Hauteur dominante}

Une étude succincte de la hauteur dominante moyenne des populations échantillonnées permet de nuancer et de compléter les conclusions concernant la croissance radiale.

La structure d'âge des populations étudiées ne permet pas toujours de déterminer la hauteur à 100 ans. Le détail pour chaque arbre des couples « hauteur / âge » est donc présenté, en séparant les quatre essences et en distinguant les types de stations par des figurés différents (fig 5). La hauteur dominante se stabilise au-delà de 100 ans, sauf pour le pin à crochets vers 150 ans. Seuls les arbres de plus de 100 ans ( 150 ans pour le pin à crochets) seront comparés.

L'épicéa atteint des hauteurs supérieures en ubac (Tar-Si-N, Mau-Ca-N) et inférieures en adret, en particulier sur calcaire (Mau$\mathrm{Ca}-\mathrm{S}$, Tar-Ca-S).

Le mélèze présente les hauteurs les plus variées. Pour le Briançonnais, elles sont maximales en ubac sur calcaire, intermédiaires en adret sur silice et minimales en ubac sur silice, alors que ce dernier type de station présente de fortes hauteurs en Tarentaise.

Le pin cembro atteint des hauteurs plus élevées en ubac (Bri-Ca-N, Bri-Si-N et Mau$\mathrm{Ca}-\mathrm{N}$ ) qu'en adret (Bel-Si-S, et Bri-Si-S dans une moindre mesure), le Briançonnais semblant être son optimum.

Enfin, les pins à crochets sont plus grands en ubac sur calcaire (Bri-Ca-N et Mau-Ca$\mathrm{N}$ ), comme l'ont déjà signalé Schueller et Rolland (1994).

Globalement, l'épicéa et le mélèze présentent des hauteurs supérieures, proches en moyenne de $20 \mathrm{~m}$, alors que les pins cembro et à crochets atteignent des hauteurs plus faibles, proches en moyenne de $15 \mathrm{~m}$. Cette observation n'a rien d'étonnant quand on sait que les bourgeons du mélèze et de l'épicéa présentent une dominance apicale que l'on ne retrouve pas chez les deux pins, au port souvent plus ramifié. La distinction de deux groupes (épicéa et pin cembro d'une part, mélèze et pin à crochets d'autre part) basée uniquement sur la croissance radiale doit donc être nuancée par l'examen de la hauteur dominante. Des quatre essences subalpines, c'est donc l'épicéa qui pousse le mieux, car sa croissance est relativement forte aussi bien en hauteur qu'en épaisseur. Viennent ensuite le mélèze, qui compense une faible croissance radiale par une assez forte croissance en hauteur, et le pin cembro pour lequel c'est l'inverse. Enfin, le pin à crochets présente une faible croissance en hauteur comme en épaisseur.

En ce qui concerne les différences entre régions, le Briançonnais, bien qu'étant la région la plus sèche dans laquelle on observe la plus faible croissance radiale, présente pour les deux espèces de pins une relativement forte croissance en hauteur.

Enfin, pour toutes les essences, les stations sur roche mère carbonatée en ubac présentent toujours des hauteurs parmi les plus élevées, alors qu'aucune distinction systématique entre les types de stations n'apparaissait au niveau de la croissance radiale.

Pour résumer schématiquement tous ces résultats, la figure 6 présente, pour chaque essence, les différentes populations étudiées en fonction de la hauteur moyenne des arbres échantillonnés et de leur âge moyen, 

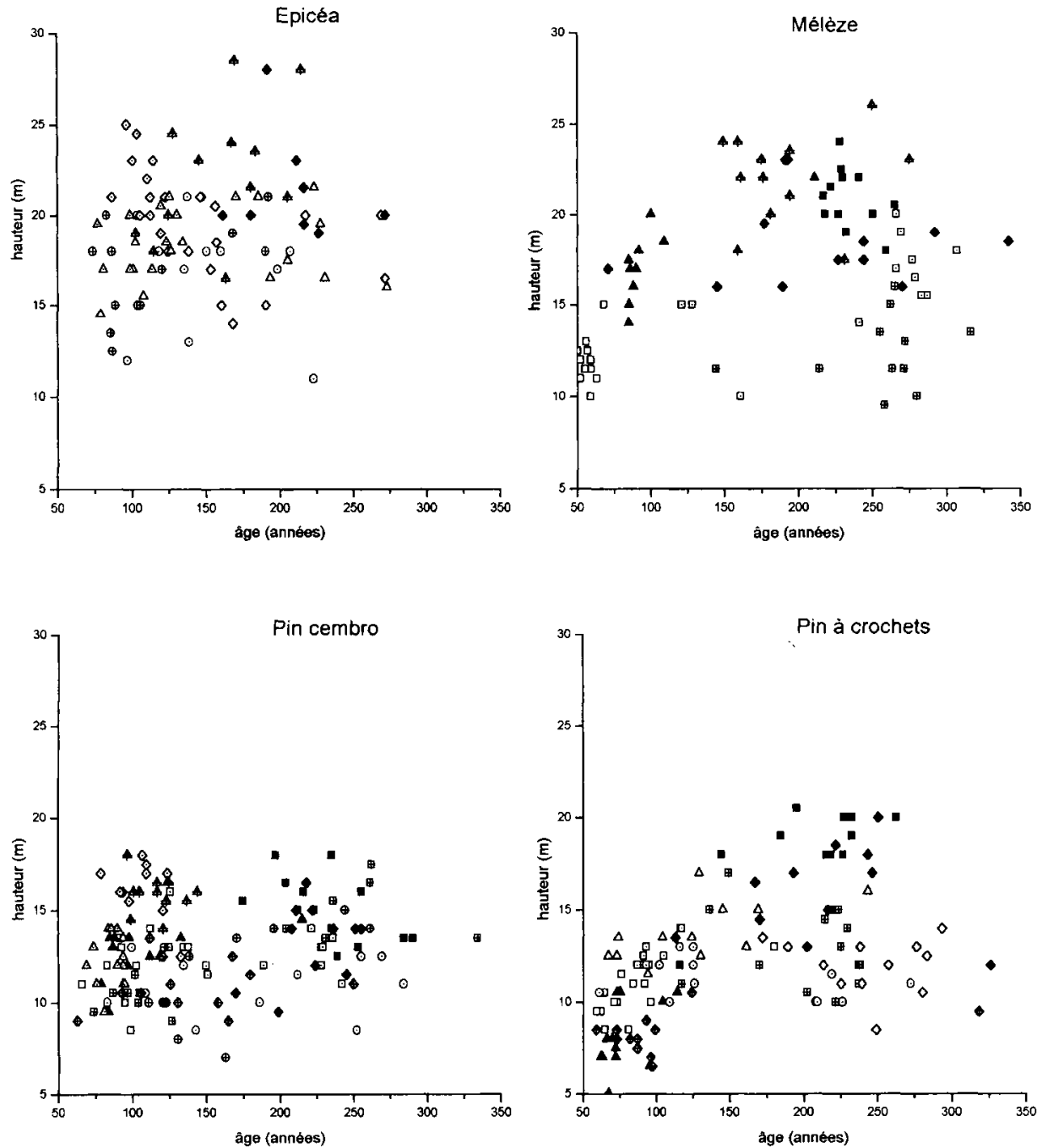
O Belledonne
$\triangle$ Moyenne Tarentaise
Haute Maurienne
$\square$ Briançonnais

\begin{tabular}{|l|c|c|}
\cline { 2 - 3 } \multicolumn{1}{c|}{} & adret & ubac \\
\hline Calcaire & & \\
\hline Silice & $\cdot$ & + \\
\hline
\end{tabular}

Fig 5. Hauteur (en m) des arbres en fonction de leur âge, pour les quatre espèces, avec un figuré différent pour chaque type de station. 

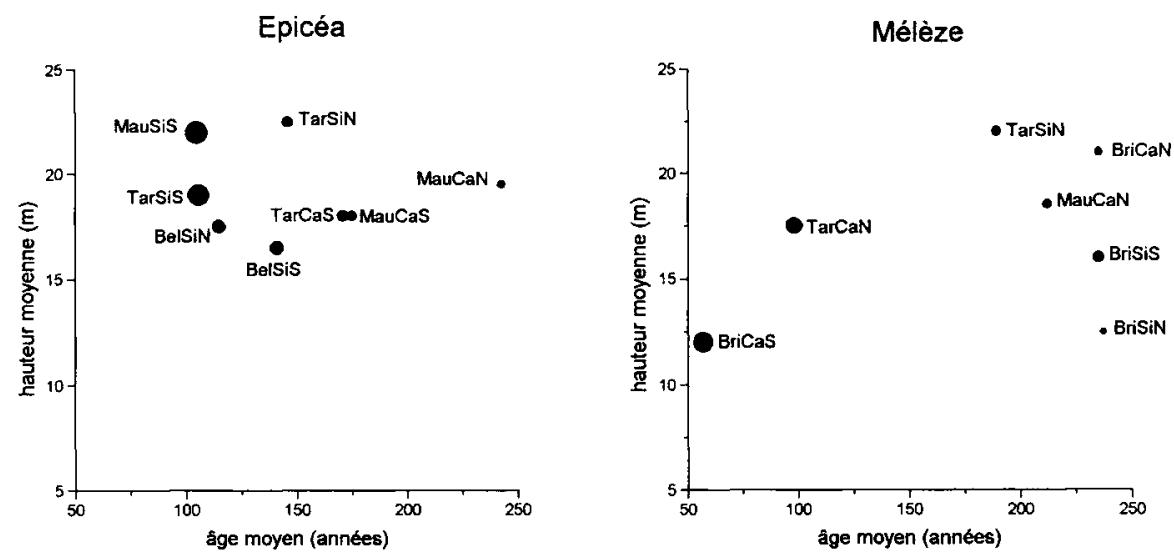

Pin Cembro

Pin à crochets
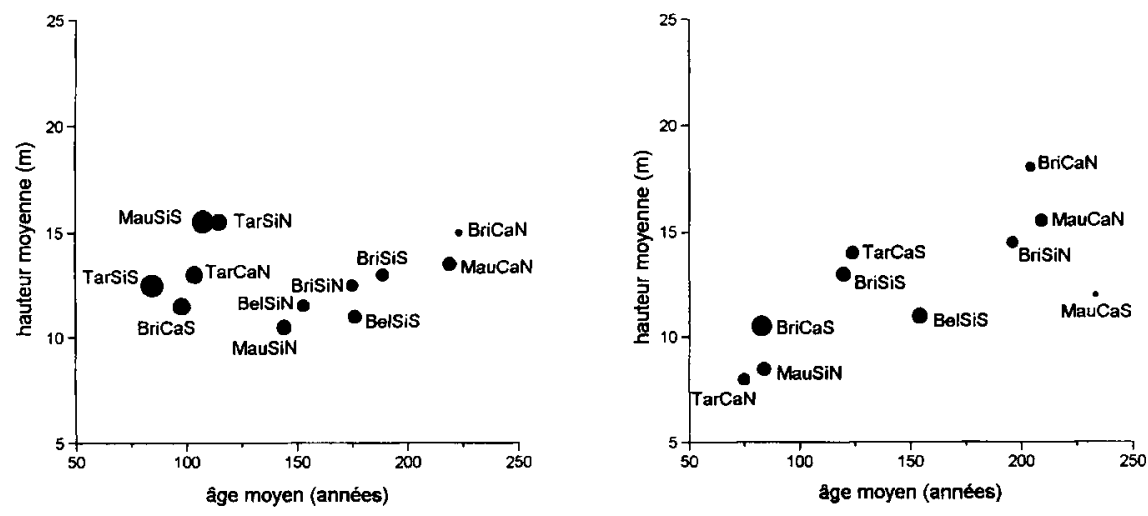

Fig 6. Hauteur dominante moyenne (en $\mathrm{m}$ ) en fonction de l'âge moyen des 37 populations, le diamètre moyen à 50 ans étant figuré par la taille des ronds noirs. Code des populations : Briançonnais (Bri), Haute-Maurienne (Mau), Moyenne-Tarentaise (Tar), Belledonne (Bel), Roche-mère silicatée (Si) ou carbonatée $(\mathrm{Ca})$, exposition Sud $(\mathrm{S})$ ou Nord $(\mathrm{N})$.

la taille des points étant proportionnelle au diamètre moyen atteint à 50 ans.

L'examen de ces diamètres à 50 ans pour les quatre essences montre que les vieilles populations ont eu une croissance beaucoup plus faible dans leur jeunesse que les populations actuellement jeunes. Ceci est moins net chez le pin à crochets mais la tendance reste la même. L'hypothèse de forêts autrefois plus denses donc à plus forte concurrence paraît peu probable en limite forestière où les recouvrements sont presque toujours faibles. En revanche, les populations à croissance rapide sont souvent 
exploitées plus précocement, ce qui explique probablement qu'on ne trouve pas de population à la fois vieille et productive.

Un climat devenu plus favorable à la croissance des arbres pourrait également en être à l'origine. En France, une augmentation récente de la croissance a déjà été mise en évidence par Bert (1992) et Becker et al (1995) pour le sapin, Belingard et Tessier (1994) pour le mélèze du Haut-Verdon, Nicolussi et al (1995) pour le pin cembro des Alpes et Badeau (1995) pour le hêtre de Lorraine.

\section{CONCLUSIONS}

Cette étude comparative de la croissance des quatre conifères constituant la limite supérieure des forêts dans les Alpes françaises permet de dégager plusieurs résultats, dont l'intérêt est principalement d'ordre sylvicole.

La comparaison des courbes de croissance radiale des 37 populations montre l'influence du climat régional et des conditions stationnelles. Dans l'ensemble, quelle que soit l'espèce, la croissance radiale apparait la plus faible dans le Briançonnais où l'aridité estivale est la plus marquée $\left(G_{e}>55^{\circ}\right)$, et la plus forte dans le massif externe de Belledonne et en Moyenne-Tarentaise. La HauteMaurienne occupe une place intermédiaire avec des croissances radiales assez faibles sur substrats calcaires mais plus élevées sur substrats silicatés. Le rôle joué par l'exposition semble dépendre de la région considérée et de la roche mère.

Ces courbes mettent également en évidence les caractéristiques de croissance propres à chaque espèce en limite altitudinale supérieure de son aire d'extension. On distingue nettement deux groupes parmi ces quatre essences:

- L'épicéa et le pin cembro qui présentent des croissances radiales relativement fortes et semblent préférer les stations sur roches silicatées et les adrets ; leurs croissances maximales sont observées en MoyenneTarentaise, les plus faibles dans le Briançonnais ( $\mathrm{pin}$ cembro) et en Haute-Maurienne sur roches carbonatées.

- Le mélèze et le pin à crochets qui présentent des croissances radiales plus faibles et plus homogènes ; les comparaisons en fonction des types de stations sont moins aisées en raison des situations non échantillonnées.

Ces résultats fournissent des courbes de référence nouvelles sur la croissance radiale de ces quatre conifères subalpins dans des conditions écologiques variées jusque là peu étudiées.

La comparaison de la croissance radiale des quatre essences tous sites confondus (surfaces terrières par âge courant) met en évidence des différences notables de rythmes de croissance montrant à quel point ces quatre conifères, même adaptés à un même type de biotope aux conditions climatiques très rudes et limitantes, peuvent utiliser des stratégies de croissance distinctes. L'épicéa présente la période de croissance soutenue la plus longue (de 80 à 150 ans environ) avant de décliner de manière très progressive; le pin cembro montre un pallier de maturité plus court et un peu plus précoce (de 75 à 125 ans); le pin à crochets atteint son maximum d'accroissement vers 50 ans et voit sa croissance en surface réduire très graduellement dès 70 ans ; enfin le mélèze présente à partir de 75 ans un pallier continu très stable qui semble se prolonger extrêmement longtemps.

L'examen des hauteurs dominantes a permis de compléter les résultats concernant la croissance radiale. Des quatre essences subalpines étudiées, c'est l'épicéa qui produit le plus de bois car sa croissance est relativement forte aussi bien en hauteur qu'en épaisseur. Viennent ensuite le mélèze, qui compense une faible croissance radiale par une assez forte croissance en hauteur, et le pin cembro pour lequel c'est l'inverse. Enfin, 
le pin à crochets présente une faible croissance dans les deux dimensions.

Cependant, il convient de relativiser la portée de l'ensemble des résultats, car d'une part ils sont basés sur un échantillonnage relativement restreint, et d'autre part la compétition inter-arbres, difficile à prendre en compte, peut interférer avec les facteurs physiques dans la croissance radiale, bien qu'il s'agisse ici de peuplements en moyenne assez peu denses. Ces résultats sont donc avant tout générateurs d'hypothèses de travail pour de futures études plus ciblées, basées alors sur des échantillonnages moins sensibles aux handicaps d'interprétation actuels.

Remerciements : Nous tenons à remercier tout particulièrement Josée Lucas pour son assistance technique lors de la mesure des 185667 largeurs de cernes, ainsi qu'Aïsha, Muriel, Esther, Vincent, Élisabeth, Clotilde, Jean-Paul, Chantal, Patty, Catherine, Éric, Gilles, Rémi, Carole et Serge pour leur aide lors du travail de terrain.

\section{RÉFÉRENCES}

Arquillière S (1986) Morphologie, croissance, reproduction végétative de l'épicéa (Picea abies L) dans une zone de combat subalpine : massif du Taillefer, Alpes dauphinoises. Thèse de troisième cycle, université Joseph-Fourier, Grenoble-I, 265 p + annexes

Badeau V (1995) Étude dendroécologique du hêtre (Fagus sylvatica L) sur les plateaux calcaires Lorrains. Influence de la gestion sylvicole. Thèse de doctorat, université Henri-Poincaré, Nancy-I. 224 $\mathrm{p}+$ annexes

Becker M (1987) Bilan de santé actuel et rétrospectif du sapin (Abies alba Mill) dans les Vosges. Étude écologique et dendrochronologique. Ann Sci For 44. $379-402$

Becker M, Bert GD, Bouchon J, Picard JF, Ulrich E (1995) Growth trends in various broadleaved and coniferous trees in Northeastern France from the mid 19 th century. Functioning and dynamics of natural and perturbed ecosystems (D Bellan, G Bonin, C Emig, eds). Technique et documentation, Lavoisier, Intercept Ltd, 1995

Bert GD (1992) Influence du climat, des facteurs stationnels et de la pollution sur la croissance et l'état sanitaire du Sapin pectiné (Abies alba Mill) dans le Jura. Étude phytoécologique el dendroécologique. Thèse de doctorat, Nancy $1,200 \mathrm{p}+$ annexes
Belingard C. Tessier L (1994) Étude dendroécologique comparée de Larix decidua Mill dans les Alpes françaises du Sud. Dendrochronologia 11, 69-78

Contini L. Lavarello Y (1982) Le pin cembro (Pinus cembra L). Répartition, écologie, sylviculture et production. Inra, Paris, $197 \mathrm{p}$

Desplanque C (1997) Dendroécologie comparée du sapin et de l'épicéa dans les Alpes internes francoitaliennes. Rôles des facteurs climatique et anthropique. Thèse, université Joseph-Fourier, Grenoble, $146 \mathrm{p}+$ annexes

Florence-Schueller J (1986) Étude dendrologique d'un mélézin du Briançonnais. Publication du laboratoire d'écologie végétale, université Joseph-Fourier, BP 53 X, 3804 I Grenoble, France

Florence-Schueller J, Rolland C (1995) Influence de l'altitude, de l'exposition et du climat sur la croissance du pin à crochets (Pinus uncinata Ram) en Cerdagne (Pyrénées-Orientales françaises). Pirineos (Jaca), 145-146, 23-34

Fourchy P (1952) Étude sur l'écologie et la sylviculture du mélèze (Larix europaea DC). Écologie du mélèze particulièrement dans les Alpes françaises. Annales de l'École nationale des eaux et forêt's 13 , 7-137

Gil-Pelegrin E, Villar-Perez L (1988) Structure of Montain Pine (Pinus uncinata Ramond) population at its upper limit in the Central Pyrénées. Pirineos (Jaca), 131

Matthewes JA (1976) "Little Ice Age" paleotemperatures from high altitude tree growth in S. Norway. Nature 264, 18 Nov 1976, 243-244

Michalet R (1991) Une approche synthétique biopédoclimatique des montagnes méditerranéennes : exemple du Maroc septentrional. Thèse de troisième cycle, UJF, Grenoble-I, 238 p + annexes

Mullenbach P (1982) Les reboisements au voisinage de la limite altitudinale de la végétation forestière (limite sylvestre). Exemple de la station du Chazelet. Premiers résultats. RFF 34, 50-71

Nicolussi K, Bortenschlager S, Körner C (1995) Increase in tree-ring width in subalpine Pinus cembra from the central Alps that may be $\mathrm{CO}_{2}$-related. Trees, Structure and Function 9, 181-189

Pache G, Michalet R, Aimé S, (1996) A seasonal application of the Gams (1932) modified Michalet (1991) method: the example of the distribution of some important forest species in the Alps. Vol. jubilaire J.L. Richard, Diss-Bot 258, J Cramer, Stuttgart, $31-54$

Petitcolas V (1993) Dendroécologie comparée de quatre espèces de conifères en limite forestière altitudinale dans le massif du Taillefer (Alpes du Nord). Rapport de DEA, UJF, Grenoble, Sept 1993,66 p + annexes

Rolland C (1996) Croissance du pin à crochets (Pinus uncinata Mill ex Mirb dans la réserve naturelle des hauts plateaux du Vercors. $R F F 48,144-152$ 
Rolland C, Schueller J (1995) Croissance comparée du pin à crochets et de l'épicéa (Pinus uncinata Ram et Picea abies Karst) sur dalle calcaire karstifiée en moyenne montagne tempérée (Vercors, France). RGA 83, 17-32

Rolland C. Schueller J ( 1996) Dendroclimatologie du pin à crochets (Pinus uncinata Mill ex Mirb) dans le Briançonnais et le Queyras en fonction des conditions stationnelles. Sc/nweiz Z Forstues 147. 351 363

Schueller J, Rolland C (1994) Influence stationnelle sur la croissance du pin à crochets (Pinus uncinata Ramond). Schweiz Z Forstwes 145, 739-755

Serre F (1978) The dendroclimatological value of the European Larch (Larix decidua $\mathrm{L}$ ) in the French Maritime Alps. Tree Ring Bulletin 38, 25-34

Siebenlist-Kerner V (1984) Der aufbau von Jahrringchronologien für Zirbelkiefer, Lärche und Fichte eines Alpinen Hochgebirgsstandortes. Dendrochronologia 2, 9-29

Tessier L (1981) Contribution dendroclimatologique à la connaissance écologique du peuplement forestier des environs des chalets de l'Orgère (parc national de la Vanoise). Tran' Sci Parc Nat Vanoise 11. 29-61

Tessier L (1986) Chronologie de mélèzes des Alpes et petit âge glaciaire. Dendrochronologia 4, 97 113

Trosset L (1984) Productivité et régénération des pessières subalpines dans les Alpes nord-occidentales. Act Biol Mont 4, 101-114

Trancuilini W (1979) Physiological ecology of the alpine timberline. Tree existence at hight altitudes with special references to the European Alps. Ec'Ological Studies, 31, Springer-Verlag, Berlin, $137 \mathrm{p}$ 